\title{
Crystal Nucleation of Colloidal Suspensions under Shear
}

\author{
Ronald Blaak, ${ }^{1}$ Stefan Auer, ${ }^{2}$ Daan Frenkel, ${ }^{3}$ and Hartmut Löwen ${ }^{1}$ \\ ${ }^{1}$ Institut für Theoretische Physik II, Heinrich-Heine-Universität, \\ Universitätsstraße 1, D-40225 Düsseldorf, Germany \\ ${ }^{2}$ Department of Chemistry, Cambridge University, \\ Lensfield Road, Cambridge, CB2 1EW, United Kingdom \\ ${ }^{3}$ FOM Institute for Atomic and Molecular Physics, \\ Kruislaan 407, 1098 SJ Amsterdam, The Netherlands
}

\begin{abstract}
We use Brownian Dynamics simulations in combination with the umbrella sampling technique to study the effect of shear flow on homogeneous crystal nucleation. We find that a homogeneous shear rate leads to a significant suppression of the crystal nucleation rate and to an increase of the size of the critical nucleus. A simple, phenomenological extension of classical nucleation theory accounts for these observations. The orientation of the crystal nucleus is tilted with respect to the shear direction.

PACS numbers: PACS: 82.70.Dd, 61.20.Ja, 81.10.Aj, 83.60.Rs
\end{abstract}

The formation of crystals in a supercooled melt is a fascinating yet complex process. It is initiated by a microscopic nucleation event. The resulting embryonic crystal then grows to macroscopic size. Understanding the principles of nucleation and growth is essential for many applications ranging from tailored protein crystallization to metallurgy [1, 2, 3]. At present, the most detailed experimental information on crystal nucleation comes from hard sphere colloids [4, 5, 6, 7]. Such suspensions are ideal to study crystal formation, as the equilibrium and transport properties of hard-sphere colloids are well understood [8]. Moreover, recent progress in computer simulations has made it possible to predict the absolute rate of crystal nucleation in colloidal suspensions 9,10$]$ and thus to compare with experiment.

In the present Letter we explore the influence of shear flow on colloidal crystal nucleation. Note that applying shear is qualitatively different from the effect of pressure, temperature or additives, as the latter affect the thermodynamic driving force for crystallization or the rate of crystal growth. In contrast, a system under shear ends up in a non-equilibrium steady state. Several experimental studies of the effect of shear on crystallization have been reported in the literature. Some of these report a shear-induced ordering of the liquid which enhances the nucleation rate 11, 12, 13, 14], while others 15, 16. report the observation of shear-induced suppression of crystallization. Both phenomena can be qualitatively understood: on the one hand, shear may induce layering in the meta-stable fluid, thus facilitating crystal nucleation. On the other hand, shear can remove matter from small crystallites and thus works against the birth of crystals. At present, it is not clear which mechanism is dominant, and under what conditions. In this Letter we combine the umbrella sampling technique from equilibrium Monte Carlo simulations with Brownian Dynamics simulations to study this non-equilibrium problem. We confirm that shear suppresses crystal nucleation, at least for small shear rates, as found by Butler and Harrowell [17], and in addition characterize the associated critical nucleus.

Below, we consider homogeneous crystal nucleation in a simple model for charge-stabilized colloidal suspensions subjected to linear shear flow. The charged colloidal particles interact via a repulsive Yukawa potential [8]

$$
V(r)=\epsilon \frac{e^{-\kappa r}}{\kappa r},
$$

where $\kappa$ is the inverse screening length and $r$ the mutual distance. The dimensionless strength of the interaction $\beta \epsilon$ has been fixed at a value $\beta \epsilon=1.48 \times 10^{4}$, where $\beta=1 /\left(k_{B} T\right)$ the inverse thermal energy and we used a cut-off at a distance $10 / \kappa$. To model the time evolution of the sheared suspension, we used Brownian Dynamics [18, 19]. In this approach, hydrodynamic interactions between the colloids are ignored. This is justified at low volume fractions of charged suspensions.

The Brownian-Dynamics equations of motion for a system in the presence of a steady shear rate $\dot{\gamma}$ are of the form

$$
\vec{r}_{i}(t+\delta t)=\vec{r}_{i}(t)+\delta t \frac{\vec{f}_{i}(t)}{\xi}+\delta \vec{r}^{G}+\delta t \dot{\gamma} y_{i}(t) \hat{x} .
$$

Here $\vec{r}_{i}(t)=\left(x_{i}(t), y_{i}(t), z_{i}(t)\right)$ is the position of the $i$ th colloidal particle at time $t$. In a small time interval $\delta t$ this particle moves under influence of the sum of the conservative forces $\vec{f}_{i}(t)$ arising from the pair interaction (11) of particle $i$ with the neighboring particles. During this motion, the solvent exerts a friction. The friction constant $\xi$ with the solvent is related to the diffusion constant $D$ by $\xi=k_{B} T / D$, while the stochastic displacements are independently draw from a Gaussian distribution with zero mean and variance $\left\langle\left(\delta r_{i \alpha}^{G}\right)^{2}\right\rangle=2 D \delta t$, where $\alpha$ stands for one of the Cartesian components. The last term in Eq. (2) represents the applied shear in the $x$-direction, 
and imposes an explicit linear flow field. For the simulations we used a cubic simulation box with 3375 particles and Lees-Edwards periodic boundary conditions 20]. The total simulation time was up to $10^{4} /\left(\kappa^{2} D\right)$ for gathering statistics. The osmotic pressure $P$ is kept at a constant value with isotropic volume moves. In practice this means that after a number of Brownian dynamics time steps the volume of the simulation box is attempted to be modified and the particles locations are scaled accordingly. The resulting difference in potential energy is used either to accept the new volume or to reject and restore the old volume and particle locations, following the rules as used in normal Monte Carlo simulation of the isobaric ensemble [18]. The results for the zero shear case show full agreement with those we obtained by equilibrium Monte Carlo simulations.

The number of particles inside the nucleus is determined with the aid of bond-orientational order parameters 21], which characterize the neighborhood of each particle. By selecting particles with a solid-like environment that are in each others neighborhood, all particles that belong to a cluster are identified.

According to the bulk phase diagram, the stable equilibrium system would be a face-centered-cubic crystalline phase 10, 22] for our parameters. The system under consideration, however, is supercooled. Hence it remains liquid, even though the solid is more stable because, unless the nucleation rates are huge [23], the simulation time required to observe spontaneous crystallization is very much longer than the duration of a run. Due to fluctuations the liquid will continuously form and dissolve small nuclei. Yet, the steady state probability $P(n)$ that a critical crystal nucleus of $n$ particles will form spontaneously is extremely small. In order to speed up this process and obtain better statistics on the cluster size distribution, we used the umbrella sampling technique 24]. The basic assumption underlying its usage is that the probability to find the system with a given cluster size is a unique function of the thermodynamic state of the system and of the shear rate. To compute the probability to find the system in an unlikely state (such as a critical nucleus), we bias the Brownian-dynamics sampling in favor of the states of interest. The actual biasing procedure is identical to the one used in (meta-stable) equilibrium studies of crystal nucleation [9], and merely works as a mathematical trick to measure the ratio of the function $P(n)$, we want to obtain, over a known and fixed probability $P_{\text {bias }}(n)$. All trajectories that are generated follow a normal path and are truncated by the bias when they deviate too much from the preferred cluster size. Rather than generating a new configuration, the last configuration is restored from which a new path is grown. Note that the trick of using umbrella sampling in a dynamical simulation is generally applicable in equilibrium and non-equilibrium situations, is not restricted to Brownian Dynamics, and enables one to obtain information on rare events.

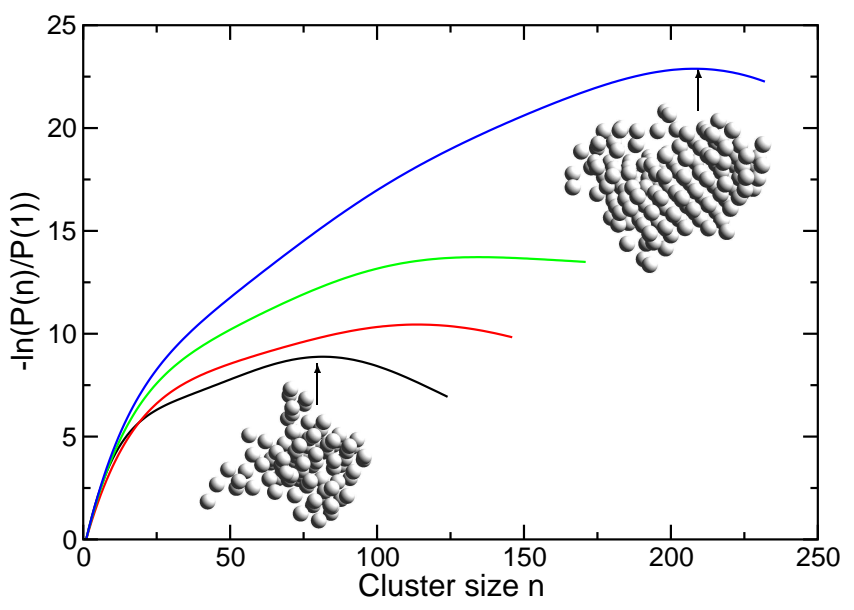

FIG. 1: Negative logarithm of the probability $P(n)$ of finding a cluster of $n$ solid-like particles normalized by $P(1)$ for pressure $\beta P / \kappa^{3}=0.24$ and different applied shear rates, from bottom to top $\dot{\gamma} /\left(\kappa^{2} D\right)=0,0.8 \times 10^{-3}, 1.6 \times 10^{-3}$, and $3.2 \times 10^{-3}$. The insets show typical snapshots of critical nuclei for the largest shear rate and the zero shear case.

After correcting for the biasing function, the cluster size distribution function is obtained. In Fig. 1 the logarithm of the probability function $P(n)$ is shown for three non-vanishing shear rates.

In the case that no shear is applied, one can relate the probability of finding a cluster of given size to the Gibbs free energy. It is therefore tempting to interpret the probability functions as shown in Fig. 1in terms of nucleation barriers [25]. Strictly speaking this is not allowed, since this idea stems from equilibrium considerations, while in the present case we treat a non-equilibrium system. However, application of statistical mechanics outside equilibrium can be useful (see e.g, 26] for an effective temperature in a sheared system) and it is a challenge to check whether and to what extent equilibrium concepts are applicable. In our case we consider the negative logarithm of the cluster size distribution function as an effective free energy.

Under this assumption a simple extension of classical nucleation theory can be made, which incorporates the shear rate. In classical nucleation theory the Gibbs free energy $\Delta G$ of a spherical nucleus of radius $R$ is given by

$$
\Delta G=-\frac{4}{3} \pi R^{3} \rho_{S}|\Delta \mu|+4 \pi R^{2} \gamma_{S L}
$$

On the one hand there is a gain in energy proportional to the volume of the nucleus due to the difference in chemical potential $\Delta \mu$ between the solid with density $\rho_{S}$ and the liquid phase. On the other hand we have a loss in energy, since an interface between the solid nucleus and surrounding liquid needs to be formed, described by $\gamma_{S L}$ the interfacial free energy.

It is reasonable to expect that for moderate shear rates the chemical potential difference $\Delta \mu$ and interfacial free 
energy $\gamma_{S L}$ will not be affected much. This would justify an expansion in powers of the shear rate for both these quantities about their equilibrium values

$$
\begin{aligned}
\Delta \mu & =\Delta \mu^{(e q)}\left(1+c_{0} \dot{\gamma}^{2}+\mathcal{O}\left(\dot{\gamma}^{4}\right)\right) \\
\gamma_{S L} & =\gamma_{S L}^{(e q)}\left(1+\kappa_{0} \dot{\gamma}^{2}+\mathcal{O}\left(\dot{\gamma}^{4}\right)\right),
\end{aligned}
$$

where due to the invariance of the shear direction only even powers in the shear rate $\dot{\gamma}$ need to be considered.

If we combine these expansions with the expression from classical theory one can easily derive expressions for $\Delta G^{*}$, the height of the nucleation barrier and $N^{*}$, the size of the critical nucleus, both of which depend quadratically on the shear rate. In Fig. 2 we show the results from our simulations where we extracted the height of the nucleation barrier for various pressures and shear rates. The dependence on the shear rate is confirmed by the parabolic fits. However, we caution the reader that this observation should not be considered as evidence that the shear rate can really be considered as a thermodynamic variable. In fact, in a recent study of the effect of shear on the location of the solid-liquid coexistence in a LennardJones system, Butler and Harrowell found that no purely thermodynamic description of the effect of shear was possible 27]. Shear directly affects the transport of particles from the solid to the liquid phase, and this effect is not thermodynamic. The expansion in Eq. (4) is simply a way to represent the effect of shear as if it were purely thermodynamic. With this caveat in mind, we continue the remainder of the discussion in the language of classical nucleation theory. We find that $N^{*}$, the number of

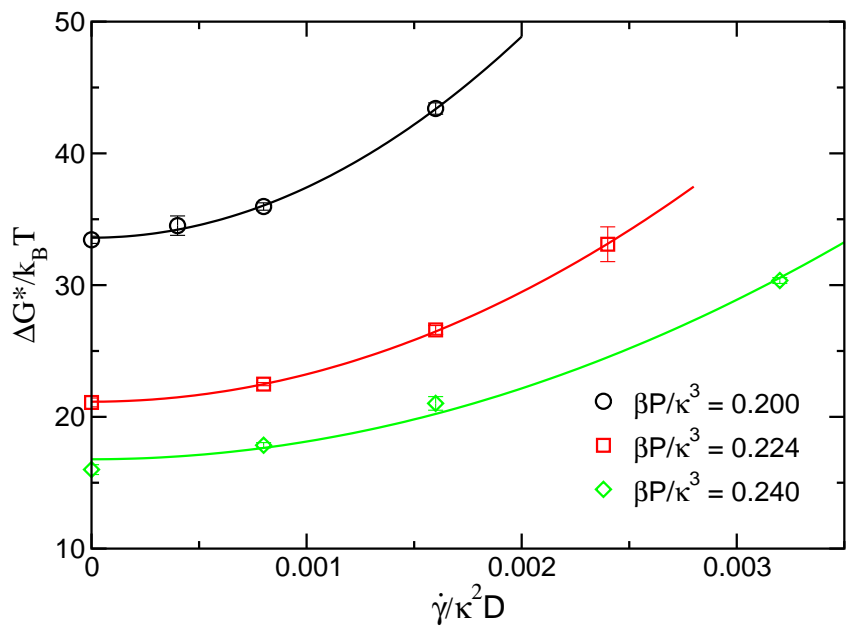

FIG. 2: The height of the nucleation barrier $\beta \Delta G^{*}$ as function of the dimensionless shear rate $\dot{\gamma} / \kappa^{2} D$ for different pressures $P$. The solid lines are parabolic fits through the data.

particles inside the critical cluster, also depends quadratically on the applied shear rate. Using the classical nucleation theory expressions $N^{*}=\left(32 \pi \gamma^{3}\right) /\left(3 \rho_{S}^{2}|\Delta \mu|^{3}\right)$ and $\Delta G^{*}=N^{*}|\Delta \mu| / 2$, we can obtain the values of the second order coefficients in Eq. (4) from a fit of the simulation data. The results are summarized in Table【 We find a negative $c_{0}$ implying a destabilization of the solid upon shear and a relatively small correction of the interfacial free energy. Both effects do not strongly depend on the pressure. Note, however, that the fits for $\Delta \mu$ and $\gamma$ do not yield a good prediction for the shape of the nucleation barrier. The shape shows deviations from the one expected by classical nucleation theory, which is due to finite size effects of the cluster.

\begin{tabular}{c|c|c|c|c}
$\beta P / \kappa^{3}$ & $\beta \Delta G^{(e q)}$ & $N^{(e q)}$ & $c_{0} D^{2} \kappa^{4}$ & $\kappa_{0} D^{2} \kappa^{4}$ \\
\hline 0.200 & 34 & 209 & $-4.8 \times 10^{4}$ & $6 . \times 10^{3}$ \\
0.224 & 21 & 133 & $-4.1 \times 10^{4}$ & $5 . \times 10^{3}$ \\
0.240 & 17 & 97 & $-3.4 \times 10^{4}$ & $4 . \times 10^{3}$
\end{tabular}

TABLE I: Numerical data for different pressures $\beta P / \kappa^{3}$ on the equilibrium barrier height $\Delta G^{(e q)}$, critical nucleus size $N^{(e q)}$, and second order corrections to the free energy difference and interfacial free energy as obtained from the fitted simulation data.

A bond order analysis shows that the structure of the nucleus is predominantly body-centered-cubic. Since small nuclei are in general neither spherical nor compact we have chosen to characterize their shape by the three principal moments of inertia. For a truly spherical nucleus these values would be identical, but since the shape of nucleus is fluctuating these moments are different. For relatively small clusters of 100 particles the ratio of the principle moments is roughly $6: 10: 12$. As the nuclei grow larger, the differences between these moments of inertia get somewhat less. Surprisingly, the imposition of shear does not influence these ratios. This leads to the conclusion that although the size of the critical nucleus increases with shear, the overall shape is hardly influenced. This is different from the radial distribution functions we measured in the liquid under shear. They become increasingly asymmetric for higher shear rates 28].

Knowledge of the eigenvectors of the inertia tensor allows us to determine its orientation. We find that the average orientation of the nucleus is weakly coupled to the direction of the applied shear. In particular, we find that the axis with the largest principal moment of inertia is, preferably in the gradient direction, in qualitative difference to a typical nearest neighbor particle cluster in a sheared fluid that prefers to be in the shear direction. The axis of the smallest principal moment of the nucleus tends to align with the vorticity direction. This alignment becomes more pronounced with increasing nucleus size and with increasing shear rate.

In Fig. 3 we show the orientation of the nucleus with respect to the shear direction. The tilt angle increases linearly with the applied shear rate $\dot{\gamma}$ and only depends weakly on the osmotic pressure. In order to improve the statistical accuracy we have averaged over all cluster sizes between $N=100$ and the critical nucleus size, $N^{*}$. The inset of Fig. 3 shows a schematic drawing of the preferred orientation of a nucleus. Note that the largest dimension of the nucleus (smallest principle moment) is preferably 
along the vorticity direction, i.e. perpendicular to the plane of drawing. Interestingly, a similar tilt occurs when vesicles with a flexible shape are exposed to a linear shear flow [29].

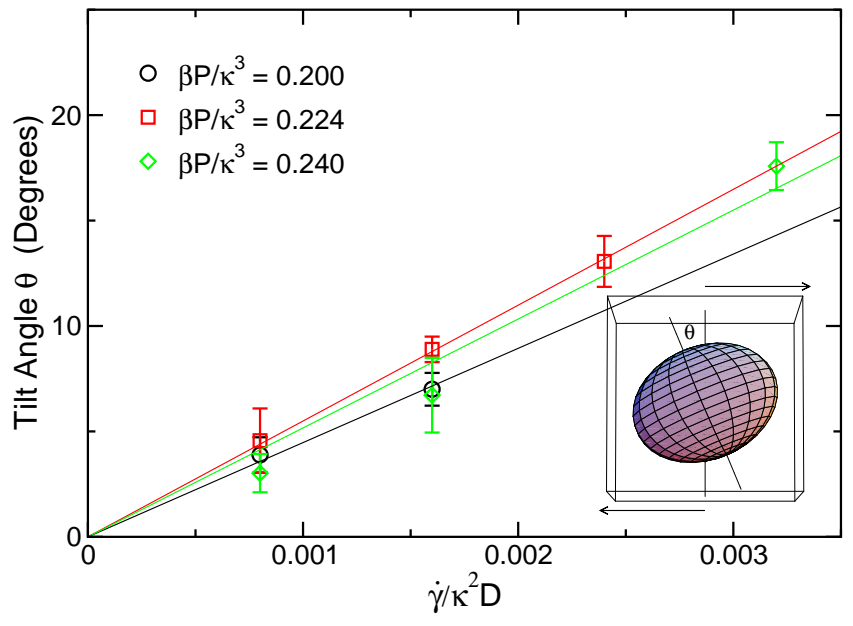

FIG. 3: The tilt angle $\theta$ of the principal moment of inertia with respect to the $y$-axis. The inset shows a schematic representation of the preferred orientation of the nucleus with respect to the shear direction indicated by the arrows.

In conclusion, we applied the combination of umbrella sampling and Brownian Dynamics simulation to the nonequilibrium problem of nucleation under shear, and found that shear suppresses nucleation and leads to a larger critical nucleus. These results can be described (but not yet understood) using a naive extension of classical nucleation theory. Most importantly, the present numerical predictions can be tested experimentally, by studying the rate of homogeneous crystal nucleation in a homogeneously sheared colloidal suspension. If nucleation were to be studied in Poiseuille flow as realized in a capillary viscometer [30], rather than in homogeneous Couette flow, we should expect crystal nuclei to appear preferentially in the middle of the flow channel.

We stress that the present findings apply to the case where the fluid is only weakly sheared, i.e. when shearinduced ordering in the liquid phase is, presumably, unimportant. We also note that the present results indicate that, during sedimentation of crystal nuclei in an otherwise stagnant solution, local shear should decrease the rate of growth of the crystallites. There may even be conditions where the competition between mass gain due to crystal growth and mass loss due to shearing, leads to the selection of one particular crystallite radius. This phenomenon should also be experimentally observable.

In this work, we ignored hydrodynamic interactions because otherwise the computational cost would have been prohibitive. This assumption, while reasonable for dilute suspensions of charged colloids, is certainly not correct in general. Finally, our method can readily be applied to other dynamical simulation methods for rare events and meta-stable systems, such as crystal nucleation in oscillatory shear [31] and heterogeneous nucleation near a system wall in a sheared suspension.

We like to thank T. Palberg, A. Van Blaaderen, G. Szamel, and S. Egelhaaf for helpful discussions. This work has been supported by DFG within subproject D1 of the SFB-TR6 program. The work of FOM Institute is financially supported by the "Nederlandse organisatie voor Wetenschappelijk Onderzoek" (NWO).

[1] K. F. Kelton, in Solid State Physics, edited by H. Ehrenreich and D. Turnbull (Academic Press, New York, 1991), vol. 45, pp. $75-178$.

[2] O. Galkin and P. G. Vekilov, Proc. Natl. Acad. Sci. USA 97, 6277 (2000).

[3] F. G. Shi, H. Y. Tong, and J. D. Ayers, Appl. Phys. Lett. 67, 350 (1995).

[4] K. Schätzel and B. J. Ackerson, Phys. Rev. E 48, 3766 (1993).

[5] J. L. Harland and W. van Megen, Phys. Rev. E 55, 3054 (1997).

[6] C. Sinn, A. Heymann, A. Stipp, and T. Palberg, Prog. Colloid Polymer Sci. 118, 266 (2001).

[7] U. Gasser et al., Science 292, 258 (2001).

[8] P. Pusey, in Liquids, Freezing and the Glass Transition, edited by J. P. Hansen, D. Levesque, and J. Zinn-Justin (North-Holland, Amsterdam, 1991), pp. 763-942.

[9] S. Auer and D. Frenkel, Nature 409, 1020 (2001); Annu. Rev. Phys. Chem. 55, 333 (2004).

[10] S. Auer and D. Frenkel, J. Phys.: Condens. Matter 14, 7667 (2002).

[11] B. J. Ackerson and P. N. Pusey, Phys. Rev. Lett. 61, 1033 (1988).

[12] Y. D. Yan, J. K. G. Dhont, C. Smits, and H. N. W. Lekkerkerker, Physica A 202, 68 (1994).

[13] M. D. Haw, W. C. K. Poon, and P. N. Pusey, Phys. Rev. E 57, 6859 (1998).

[14] R. M. Amos et al., Phys. Rev. E 61, 2929 (2000).

[15] T. Palberg, W. Mönch, J. Schwarz, and P. Leiderer, J. Chem. Phys. 102, 5082 (1995).

[16] T. Okubo and H. Ishiki, J. Colloid and Interface Science 211, 151 (1999).

[17] S. Butler and P. Harrowell, Phys. Rev. E 52, 6424 (1995).

[18] M. P. Allen and D. J. Tildesley, Computer simulations of liquids (Oxford University Press, Oxford, 1987).

[19] J. Chakrabarti, A. K. Sood, and H. R. Krishnamurthy, Phys. Rev. E 50, R3326 (1994).

[20] A. W. Lees and S. F. Edwards, J. Phys. C 5, 1921 (1972).

[21] P. J. Steinhardt, D. R. Nelson, and M. Ronchetti, Phys. Rev. B 28, 784 (1983).

[22] S. Hamaguchi, R. T. Farouki, and D. H. E. Dubin, Phys. Rev. E 56, 4671 (1997).

[23] B. O'Malley and I. Snook, Phys. Rev. Lett. 90, 085702 (2003).

[24] G. M. Torrie and J. P. Valleau, Chem. Phys. Lett. 28, 578 (1974).

[25] S. Auer and D. Frenkel, J. Chem. Phys. 120, 3015 (2004).

[26] I. K. Ono et al., Phys. Rev. Lett. 89, 095703 (2002).

[27] S. Butler and P. Harrowell, J. Chem. Phys. 118, 4115 (2003).

[28] R. Blaak, S. Auer, D. Frenkel, and H. Löwen, J. Phys.: Condens. Matter (to be published 2004).

[29] M. Abkarian, C. Lartigue, and A. Viallat, Phys. Rev. Lett. 88, 068103 (2002). 
[30] T. Palberg and M. Würth, J.Phys. I France 6, 237 (1996).

[31] W. Xue and G. S. Grest, Phys. Rev. A 40, R1709 (1989). 